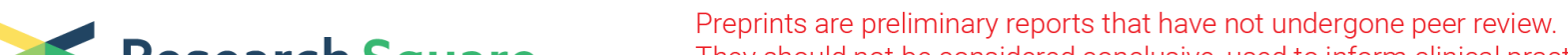 $\begin{array}{ll}\text { Research Square } & \text { They should not be considered conclusive, used to inform clinical practice, } \\ \text { or referenced by the media as validated information. }\end{array}$
}

\section{Antimalarial Activity of Brusatol Against Plasmodium Berghei Infected Mice and the Mechanism Revealed by Whole Transcriptome Sequencing (RNA-Seq) Analysis}

\section{Yixin Ren}

Dalian Medical University

\section{Shanshan Xu}

Dalian Medical University

\section{Yuan Gao}

Dalian Medical University

\section{Yufeng Chen}

Dalian Center for Disease Control and Prevention

\section{Xiaodong Dai}

Dalian Medical University

\section{Lei Shi}

Dalian Medical University

\section{Feng Zhao}

Dalian Medical University

\section{Yuanhua Qin ( $\nabla 54386546 @ q q . c o m$ )}

Dalian Medical University https://orcid.org/0000-0002-9405-3159

\section{Research Article}

Keywords: Antimalarial drugs, Plasmodium berghei, Brusatol, Brucea Javanica

Posted Date: May 4th, 2021

DOl: https://doi.org/10.21203/rs.3.rs-429136/v1

License: (c) (i) This work is licensed under a Creative Commons Attribution 4.0 International License. Read Full License 


\section{Abstract}

\section{Background}

Recently, artemsinin-resistant malaria strains and clinical cases have appeared in Southeast Asia. Reportedly, there are malaria mutants in Africa that are resistant to artemisinin and its derivatives. Thus, it's imminent to develop new antimalarial drugs. Brucea javanica is an effective antimalarial drug recorded in Chinese traditional medicine, which has been widely used in the folk for hundreds of years. Brusatol is the main active constituent of Brucea Javanica, thus we studied the effects of brusatol on prevention of malaria infection in vivo.

\section{Methods}

To determine the antiplasmodial activity of brusatol, a four-day suppressive test was used by dividing 56 mice into 7 groups of 8 mice each and given $4 \mathrm{mg} / \mathrm{kg}, 3 \mathrm{mg} / \mathrm{kg}, 2 \mathrm{mg} / \mathrm{kg}, 1 \mathrm{mg} / \mathrm{kg}, 0.5 \mathrm{mg} / \mathrm{kg}$ of brusatol, the standard drug ((artesunate of $140 \mathrm{mg} / \mathrm{kg}$ ) and the vehicle (normal saline).

The best effective dose was used in the following test. The effects of brusatol to plasmodium berghei transcription were tested through RNA-seq and the results were confirmed by RT-qPCR. We also explored the expression of TNF - $\mathrm{a}$, IFN- $y$, IL-4, IL-12 to evaluate antimalarial mechanism of brusatol to host by ELISA.

\section{Results}

The results showed that brusatol effectively inhibited plasmodium berghei infection, the best effective dose was $2 \mathrm{mg} / \mathrm{kg}$, and the side effects of brusatol to liver and kidney were slight and reversible. The expressions of GSK3 $\beta$, ATP6A, ATP6B, ATP6M, MSP-2, EMP1, CTCS in plasmodium were significantly lower after brusatol treatment compared with control, while the expression of AMA-1 was significantly increased. The serum concentrations of IFN- - , TNF- $\alpha$ and IL-4 in artesunate and brusatol group decreased significantly compared with the control group, while there was no statistical difference of the serum concentrations of IL-12.

\section{Conclusions}

Taken together, these results demonstrated brusatol could be a priority candidate for antimalarial medicine development.

\section{Introduction}

Malaria remains a highly fatal infectious disease that seriously threatens human beings' health. According to the World Health Organization (WHO) report, an estimate of 229 million malaria cases have been reported, including 409,000 deaths worldwide in 2019. Most cases were reported in the aeras of subSaharan Africa, accounting for about $93.4 \%$ of total malaria cases [1]. Artemisinin-based combination 
therapies (ACTs) have been widely used and gained great achievement in the control of malaria in the past decades. However, in 2008, artemisinin-resistant malaria strains were firstly emerged in western Cambodia and prevalent along the Thai-Cambodia border [2], then artemisinin-resistant cases were reported in Southeast Asia areas, making the the prevention of malaria increasingly serious [3-6]. Recently, a collaborative study from Pasteur Institute, Rwanda Biomedical Centre, Columbia University and the World Health Organization identified and confirmed the malaria parasite was mutated in Africa that is resistant to aremisinin and its derivatives [7]. Thus, to seek for new and effective antimalarial drugs is urgent.

Brucea javanica is an effective antimalarial drug recorded in Chinese traditional medicine. Researchers from various countries have screened a large number of herbal medicines for their antimalarial effects and conducted in vitro and in vivo experiments. The in vitro and in vivo experiments showed Brucea javanica possessed strong antimalarial effect, similar with the effects of chloroquine in vitro [8-13]. Brusatol is the main active constituent of Brucea Javanica, and the median lethal dose (LD50) of Brucea Javanica $(7.58 \mathrm{ng} / \mathrm{ml})$ is equivalent to the antimalarial effect of chloroquine [9]. The mutation of Kelch propeller protein encoded by $\mathrm{K} 13$ gene of $P$. falciparum was related to its artemisinin- resistance [14]. Kelch propeller protein is the main protein product of $\mathrm{K} 13$ gene, which is homologous with human Keap1 protein. Current studies have confirmed that Keap1, a homologue of Kelch propeller protein, is a specific receptor of Nrf2 $[15,16]$. Brusatol is a natural inhibitor of Nrf2, which may block the activity of Nrf2 to inhibit malaria and sensitize artemisinin-resistant plasmodium to the drug [17].

This study investigated the antimalarial effect of brusatol and its toxicity through in vivo assay, and explore the antimalarial mechanism of brusatol through the RNA-seq, RT-qPCR and ELISA assays. These studies can provide the evidences for the development of brusatol as a new antimalarial drug.

\section{Materials And Methods}

\section{Mice and parasites}

BALB/c mice, female, 6-8 weeks old (weighted 18-25g) were purchased from the Animal Experimental Center of Dalian Medical University. The animals were housed in plastic cages at room temperature and were handled according to international guidelines for use and maintenance of experimental animals (OECD/OCDE, 2008). Plasmodium berghei ANKA strain was obtained from the Department of Parasitology, Dalian Medical University. Whole blood was taken from the infected mouse and diluted in normal saline, every $0.2 \mathrm{ml}$ of the aliquot contained about $1 \times 10^{7}$ infected RBCs.

\section{Drugs and chemicals}

Artesunate powder was bought from Guilin South Medicine Co. Ltd. The powder was dissolved in 5\% sodium bicarbonate, and finally diluted to $14 \mathrm{mg} / \mathrm{ml}$ with normal saline, which was used as the artesunate injection. Brusatol crystals were obtained from Beijing Solarbio Science \& Technology Co. Ltd. It was dissolved by dimethyl sulfoxide (DMSO), and finally diluted to $0.2 \mathrm{mg} / \mathrm{ml}$ with normal saline, which 
was used as the brusatol injection. Artesunate injection was placed for no more than $1 \mathrm{~h}$ after preparation; Brusatol injection was stored at $4{ }^{\circ} \mathrm{C}$ away from light, no more than 4 days.

\section{Four-day suppressive test}

$56 \mathrm{BALB} / \mathrm{c}$ mice were inoculated intraperitoneally(i.p.) with $0.2 \mathrm{ml}$ infected blood described as before (contained about $1 \times 10^{7}$ P. berghei infected RBCs), and they were divided into 7 groups of 8 mice. According to Peter's four-day suppressive test [18], at 4 hours after $P$. berghei injection, Mice were given $4 \mathrm{mg} / \mathrm{kg}, 3 \mathrm{mg} / \mathrm{kg}, 2 \mathrm{mg} / \mathrm{kg}, 1 \mathrm{mg} / \mathrm{kg}, 0.5 \mathrm{mg} / \mathrm{kg}$ of brusatol, the standard drug (artesunate of $140 \mathrm{mg} / \mathrm{kg}$ ) and the vehicle (normal saline) for four consecutive days (Day0 to Day3). Blood was extracted from mice tails top on Day4, and observed under oil immersion lens after Wright's stain.

\section{Blood routine examination and hepatic and renal toxicity study}

48 BALB/c mice were randomly divided into 3 groups of 16 mice. Mice were injected $140 \mathrm{mg} / \mathrm{kg}$ artesunate, $2 \mathrm{mg} / \mathrm{kg}$ brusatol and normal saline as the control for 4 days (Day0-Day3). 8 mice were randomly selected from each group on Day4. Their eyeball blood was extracted and anti-coagulated with EDTA-K2. Gently mix the blood and anticoagulation before hematology analyzer (HF-3800) analyzes the whole blood cells. The upper serum was collected after centrifuging at $4{ }^{\circ} \mathrm{C}, 2000 \mathrm{~g}$ for $5 \mathrm{~min}$. The concentration of the serum AST, ALT, TP, ALB, GLOB, CREA and UREA were measured. The above steps were repeated on $\mathrm{D} 28$ with the remaining mice.

\section{ELISA to detect serum cytokines level}

$42 \mathrm{BALB} / \mathrm{c}$ mice were inoculated intraperitoneally(i.p.) with $0.2 \mathrm{ml}$ infected blood (contained about $1 \times 10^{7}$ $P$. berghei infected RBCs), and they were divided into 3 groups of 14 mice. At 4 hours after $P$. berghei injection, mice were given $2 \mathrm{mg} / \mathrm{kg}$ brusatol, the standard drug (artesunate of $140 \mathrm{mg} / \mathrm{kg}$ ) and the positive control (normal saline) for four consecutive days (Day0 to Day3). Meanwhile, 14 mice without infection were inoculated intraperitoneally with normal saline from Day0-Day3 as the negative control. Eyeball blood was extracted from all mice and anti-coagulated with EDTA-K2 on Day4.

Among 3 groups of 14 blood samples, 3 groups of 8 blood samples were centrifuged at $4^{\circ} \mathrm{C}, 2000 \mathrm{~g}$ for $5 \mathrm{~min}$ for the upper serum. The serum was stored at $-20^{\circ} \mathrm{C}$ for later ELISA. Other blood samples were stored at $-80^{\circ} \mathrm{C}$ for RNA-seq and Quantitative real-time RT PCR(RT-qPCR) analysis.

\section{ELISA to detect serum cytokines level}

Serum levels of IFN-y,TNF-aIIL-4 and IL-12 were measured by ELISA (Elabscience Biotechnology Co., Ltd). The serum was incubated in the assay plates at $37^{\circ} \mathrm{C}$ for $30 \mathrm{~min}$. After washing for five times, the HRPconjugated reagent was added and incubated at $37^{\circ} \mathrm{C}$ for $30 \mathrm{~min}$. After another washing for five times, samples were incubated with chromogen solution $A$ and $B$ at $37^{\circ} \mathrm{C}$ for 10 min. Finally, the reactions were analyzed at $490 \mathrm{~nm}$ in a microplate reader.

\section{RNA-seq analysis}


The fragments per kilobase of transcript per million mapped reads (FPKM) method were used to calculate the mRNA levels of genes. Noiseq method (probability $\geq 0.8 \& \log 2$ fold-change $>1$ ) was used to screen DEGs. All these DEGs were mapped to the database of KEGG and GO for pathway and GO enrichment analysis.

\section{RT-qPCR validation}

8 candidate genes were randomly selected for RT-qPCR validation. Total RNA was extracted from the $P$. berghei-infected blood samples using RNAiso Plus (Takara, Japan) and reverse transcribed into cDNA using PrimeScript RT reagent kit (Takara, Japan). Quantitative real-time RT PCR (RT-qPCR) was performed with an Mx3000P QPCR system (Agilent Technologies, USA) using SYBR Premix DimerEraser (Takara, Japan). Seryl was selected as housekeeping genes to determine their transcription stability. PCR cycling parameters ( 30 cycles) were set as denaturation $\left(95^{\circ} \mathrm{C}, 30 \mathrm{~s}\right)$, annealing $\left(59^{\circ} \mathrm{C}, 30 \mathrm{~s}\right)$, and extension $\left(72^{\circ} \mathrm{C}, 1 \mathrm{~min}\right)$. The primer sequences used for qPCR are presented in Table.1. The data are expressed as $2^{-\Delta \Delta \mathrm{Ct}}$.

Data were expressed as mean \pm S.E.M. All statistical analyses were done by GraphPad Prism (version 8.0; GraphPad Software). The difference in mRNA expression level between the control and brusatol group were conducted by using the unpaired T-test. $p<0.05$ was considered as statistically significant.

Table 1

Primer sequences used for RT-qPCR analysis.

\begin{tabular}{|c|c|c|}
\hline Gene & Forward sequence (5'-3') & Reverse primer (5'-3') \\
\hline ATP6A & AATATATGTTGGGTGTGGTGAAAGAG & GCTGCGACCGGCATATTAGA \\
\hline ATP6B & TGGAAATCCAATTAACCCACAAT & GCACCTATCTCATTATGTGGCAAA \\
\hline ATP6M & AGCTCAAAATGCTAAACAAGAAGCT & AAAGAAAAAAGCCCСССТTTAA \\
\hline MSP-2 & TGATGGTAATGGTGCAGATGCT & GTTTCGGCATTTTTATGATTTGG \\
\hline AMA-1 & TGTTGTATGTAGCAGCCCAAGAA & CCCAATCATCACGCAAATTTT \\
\hline EMP1 & AAAGAACAAGACCATAATAAACCATACG & ACCCTTTGGCATTTGAGGATT \\
\hline GSK3 $\beta$ & ATCTGGGCAAAGGAGCGTTT & GAAAATATTGGATATCCCAGAACCA \\
\hline CTCS & AATTCGGAAAACTTAAGACCTGAGTT & CGGCAAGATAAGTCCAAATACGA \\
\hline
\end{tabular}

\section{Results}




\section{$2 \mathrm{mg} / \mathrm{kg}$ brusatol effectively inhibit the growth of P. berghei}

Compared to the control, brusatol reduced parasitemia significantly. $2 \mathrm{mg} / \mathrm{kg}$ was the most ideal therapeutic dose of brusatol to treat the $P$. berghei infected mice. At $2 \mathrm{mg} / \mathrm{kg}$ dose, the antimalarial effect of brusatol on $P$. berghei was similar with $140 \mathrm{mg} / \mathrm{kg}$ artesunate at the concentration of parasitemia lower than 5\% (Fig. 1A, B). Then, we tested the effect of brusatol on malaria anemia, and found that brusatol $(2 \mathrm{mg} / \mathrm{kg})$ can improve the anemia caused by malaria as well as artesunate.

\section{The hepatic and renal damage caused by brusatol is reversible}

On Day4, compared with the control and artesunate group, the AST, ALT, CREA and UREA in serum were significantly increased in the brusatol group, and the TP, ALB and GLOB were significantly decreased in the brusatol group. On Day28 after treatment, there was no statistical difference of these markers between brusatol group and control group or artesunate group (Fig. 2).

On Day4, brusatol treatment influenced the liver and kidney function of mice, however, when the drug treatment was stopped for 25 days (from Day4 to Day28), all serum concentrations of markers revered to normal levels, showing the abnormal of liver and kidney function caused by brusatol was reversible after stopping the injections of brusatol.

\section{Brusatol regulated immune cytokines to inhibit the P. berghei infection}

After infection, $P$. berghei stimulated the host immune system, causing the serum IFN- - , TNF- $\mathrm{a}$, IL-4 and IL-12 significantly increased in $P$. berghei-infected mice. Compared with the control group, the serum concentrations of IFN- $\gamma$, TNF- $a$ and IL- 4 in artesunate and brusatol group decreased significantly, while there was no statistical difference of the serum concentrations of IL-12. (Fig. 3)

The results showed that brusatol could play function against $P$. berghei by regulating the expression of host IFN-y, TNF- $a$ and IL-4, whose efficiency was almost equal to artesunate.

\section{RNA-seq analysis and validation of DEGs}

Our study identified 812 unigenes as DEGs between the control and brusatol group, including 388 upregulated and 424 down-regulated genes (BioProject ID PRJNA699138, Fig. 4A). The results were further validated using the Morpheus online tool, and the DEGs are presented in a hierarchical clustering heat map (Fig. 4B, C). GO and KEGG databases were used to identify the function of DEGs. We found that brusatol had a wide range of effects on various biological pathways of $P$. berghei, and the distribution of DEGs in 'Biological Process', 'Cellular Component' and 'Molecular Function' was as shown in the figure (Fig. 4B). The majority of the DEGs were demonstrated to be significantly enriched in BPs, CCs, MFs, 
including the 'cellular process' and 'metabolic process' in BPs, the 'membrane' and 'membrane part' in CCs,and the 'catalytic activity' and 'binding' in MFs. KEGG pathway analysis demonstrated that the DEGs were enriched in 'Transport and catabolism', 'cell growth and death', 'signal transduction', 'folding, sorting and degradation', 'energy metabolism' and 'nucleotide metabolism' (Fig. 4C).

8 DEGs (1 up- and 7 down-regulated DEGs), ATP6A, ATP6B, ATP6M, MSP-2, AMA-1, GSK3 $\beta$, EMP1, CTCS were selected for RT-qPCR analysis. The expression of these eight genes was demonstrated to be significantly lower after brusatol treatment compared with control except the expression of AMA-1 (all $P<$ 0.01 ; Fig. $5 \mathrm{~A}$ ). Consistent with the results of the database analysis, mRNA expression of them was significantly lower in brusatol groups compared with control groups (7 of $8 P<0.05$; Fig. 5B), while the expression of AMA-1 was significantly increased compared with control groups $(P<0.05$; Fig. 5B).

\section{Discussion}

Malaria is still one of the most infectious diseases threatening the people's health all over the world. ACTs, as the first-line antimalarial drug, has shown a resistant trend in the treatment effect of malaria in Southeast Asia recently. The fundamental cause is the emergence of both artemisinin and the combined drug-piperaquine resistance. The thorough solution to the resistant plasmodium strains is to develop new antimalarial drugs. Brucea Javanica is an effective antimalarial herb medicine recorded in traditional Chinese medicine, and the brusatol also exhibited antimalarial effect.

We studied the antimalarial effect of different concentrations of brusatol in parasitemia of $P$. bergheiinfected BALB/c mice and compared it with artesunate $(140 \mathrm{mg} / \mathrm{kg})$. We found that $2 \mathrm{mg} / \mathrm{kg}$ of brusatol was the lowest concentration to achieve the ideal therapeutic effect. At this dose, brusatol treatment could control the parasitemia under $5 \%$, which had the same antimalarial effect as artesunate of 140 $\mathrm{mg} / \mathrm{kg}$. It is proved that brusatol can play an effective role in the treatment of $P$. berghei at the dose of 2 $\mathrm{mg} / \mathrm{kg}$.

To explore the toxic effects of brusatol on the liver and kidney function in mice, we simulated the treatment process but without $P$. berghei infection, and then measured the serum concentrations of AST, ALT, TP, ALB, GLOB, CREA and UREA on Day4 and Day28 respectively. AST, ALT, TP, ALB and GLOB are serum markers of liver function; UREA and CREA are serum markers of renal function. On Day4, compared with the control and artesunate group, in the brusatol group, the serum concentrations of AST, ALT, CREA and UREA significantly increased, and the serum concentrations of TP, ALB and GLOB significantly decreased, which indicated that the liver and kidney functions of brusatol group mice were abnormal. Compared with the artesunate group, the concentration changes of serum markers in brusatol group were more significant, which demonstrated that the effect of brusatol on liver and kidney function of mice was more than that of artesunate. To further observation that whether the liver and kidney damage caused by brusatol was reversible, we measured the serum markers above on Day28 after conventional brusatol treatment again. Compared with the control group, the levels of all serum markers returned to normal degree, indicating when the drug has been stopped for a period of time (on Day28), the 
liver and kidney function can return to normal condition. The damage of liver and kidney function caused by brusatol is slight and reversible.

There are significant changes in a variety of cytokines in mice infected with malaria, in which the increase of TNF-a, IFN-y, IL-4 and IL-12 are significant, closely related to the host immune mechanism against plasmodium [19]. IFN- $\gamma$ is mainly produced by activated T cells and NK cells, and activates erythrocytic specific cells and antibody-dependent cells to kill the malaria parasites in the erythrocytic stage [20-23]. TNF-a is released by monocytes and macrophages. The level of TNF-a in cerebral malaria resistant mice infected with $P$. berghei is higher than that in susceptible mice [24]; the epidemiological studies also suggest that there is a potential protective effect of TNF-a on malaria infection [25]. IL-4 is mainly produced by Th2 cells, activated basophils and mast cells. By inhibiting the activity of monocytes and macrophages, IL-4 weakens their killing effects on the malaria parasites in the erythrocytic stage, and its protection mechanism is also related to its inhibition or down-regulation of inflammatory cytokines secretion $[24,26]$. IL-12 is produced by activated macrophages, B cells, DC cells, etc. It can help the host to eliminate the malaria parasites by enhancing the killing activity of NK cells and promoting the production of cytotoxic $\mathrm{CD} 8^{+} \mathrm{T}$ cells. It also plays a specific role in eliminating the malaria parasites by regulating the production of IFN- $\gamma$ and TNF-a by T cells and NK cells to induce Th1 cell immunity $[27,28]$. It is suggested that TNF-a, IFN- $y$, IL-4, and IL-12, the four cytokines can reflect the severity of hosts' malaria, so we studied the serum changes of these cytokines after treatment with brusatol. It was found that the concentrations of TNF- $a$, IFN- $y, \mathrm{IL}-4$ and IL-12 in the serum of mice infected with $P$. berghei were significantly increased, which were well agreed with that reported in the literature. However, the concentrations of TNF-a, IFN- $y$ and IL-4 in the serum of mice treated with brusatol were significantly decreased $(P<0.01)$, while there was no statistical difference of the serum concentrations of IL-12. It is suggested that brusatol can exert its antimalarial activity by regulating the expression of TNF-a, IFN- $\gamma$, IL4 , and affecting the functions of host T cells, NK cells, monocytes, macrophages and erythrocytes.

To study the antimalarial mechanism of brusatol, we screened 812 DEGs, including 388 up-regulated genes and 424 down-regulated genes, by high throughput screening (HTS) of $P$. berghei in blood samples of mice in the control group and brusatol group. According to Go analysis, DEGs were demonstrated to be significantly enriched in "cellular process", "metabolic process", "membrane", "catalytic activity" and "binding"; KEGG pathway analyses show that DEGs were demonstrated to be associated with "Transport and catabolism", "cell growth and death", "signal transduction", "folding, sorting and degradation", "energy metabolism" and "nucleotide metabolism". The results showed that brusatol could help the host eradicating the malaria parasites by influencing the metabolism process, the function of the cell membrane and receptor, the catalytic process, and so on. Among these DEGs, we screened several DEGs closely related to the growth, metabolism and invasion of P. berghei: ATP6A, ATP6B, ATP6M, MSP-2, AMA-1, GSK3ß, EMP1, CTCS. The results of the RT-qPCR analysis were consistent with the result of HTS.

Protein kinases related to growth and metabolism of $P$. berghei: Glycogen synthase kinase $3 \beta$ (GSK3 $\beta$ ) is a Ser/ Thr protein kinase commonly distributed in eukaryotic cells, activated by tyrosine phosphorylation and inhibited by serine phosphorylation. Plasmodium falciparum Glycogen synthase kinase 3 (PfGSK3) 
was confirmed to be necessary for the growth of $P$. falciparum, so it is considered to be an important target of new anti-malarial drugs. Recently, sensitive PfGSK3 inhibitors were considered as potential new anti-malarial drugs [29]. Compared with the control group, the expression of GSK3 $\beta$ in $P$. berghei treated with brusatol decreased significantly $(P<0.01)$, indicating that GSK3 $\beta$ is an important target of brusatol in anti-malarial treatment. Plasmodium falciparum calcium ATPase 6 (PfATP6) is a kind of Sarco/endoplasmic reticulum $\mathrm{Ca}^{2+}$-ATPase. It regulates the intracellular calcium concentration of $P$. falciparum by consuming ATP, thus maintaining the stability of calcium concentration in the malaria parasites. PfATP6 was known as one of the effective targets of artesunate against malaria [30]. Artesunate and its derivatives inhibit PfATP6, which leads to an increase of intracellular calcium concentration and plays a role in eradicating $P$. falciparum. The malaria Parasites also showed drug resistance through PfATP6 gene mutation [31, 32]. After treatment with brusatol, the expression of several typical ATP6 protein kinases: ATP6A,ATP6B, ATP6M in $P$. berghei decreased significantly $(P<0.01)$, indicating that brusatol can kill the malaria parasites by inhibiting the expression of ATP6, and it may be a new anti-malarial drug to solve artesunate resistance.

3 proteins among them, MSP-2, EMP1 and AMA-1, are closely related to the invasion and immune escape of $P$. berghei. Glycosylphosphatidylinositol anchored protein (GPI-AP) MSP-2, is the second abundant protein on the merozoite surface of $P$. falciparum. It may participate in the adhesion process of the malaria parasites to host red blood cells and play an important role in its invasion to red blood cells [33]. MSP-2 is a potential target for anti-malaria vaccines or drugs. The candidate vaccine based on MSP-2 had an obvious anti-malaria effect on the invasion of $P$. falciparum into red blood cells $[34,35]$. After treatment with brusatol, the expression of MSP-2 of $P$. berghei in mice decreased significantly $(P<0.01)$, indicating that brusatol can inhibit the invasion of $P$. berghei into the host red blood cells by reducing the expression of MSP-2. PfEMP1 is a variable antigen expressed by $P$. falciparum, which exists on the surface of infected host red blood cells and mediates the combination of infected red blood cells and vascular endothelial cells so that the malaria parasites can avoid spleen clearance [36, 37]. Also, PfEMP1 regulated the host's immune response by binding the CD36 receptor on antigen- presenting cells, while inhibited the production of IFN- $\gamma$ in human peripheral blood mononuclear cells (PBMCs) in the early stage of $P$. falciparum infection [38]. After treatment with brusatol, the expression of $P$. berghei EMP1 in mice increased significantly $(P<0.01)$, which indicated that brusatol could help host immune system recognize malaria parasites by reducing the expression of EMP1, thus promoting host clearance of malaria parasites. The Plasmodium falciparum apical membrane antigen 1 (PfAMA-1) is synthesized in the erythrocytic stage of $P$. falciparum. In recent years, studies have reported AMA-1 of Plasmodium species featured functional conservation, providing the theories foundation for the development of cross-species inhibitors against malaria [39]. After treatment with brusatol, the expression of $P$. berghei AMA-1 in mice increased significantly $(P<0.01)$, which indicated that brusatol could promote the recognition and immune clearance of the host to the malaria parasites by increasing the expression of the parasite AMA1. After treatment with brusatol, the expression of CTCS of $P$. berghei also decreased significantly, while the results of RT-qPCR demonstrated that its expression was very low, sometimes even could not be detected. It is speculated that this protein is an important target of brusatol against malaria. However, 
there are few studies on CTCS, and the role of CTCS in the parasites' growth, development, or invasion is still unclear, which needs further study.

Besides, after treatment with brusatol, the expression of many genes in $P$. berghei increased or decreased significantly, which may also be the target of brusatol against malaria, but their specific mechanisms are still unknown. Further studies will be needed. As more and more malaria parasites show resistance to artesunate and its derivatives, protein kinases that regulate parasites growth and differentiation have become new targets of antimalarial drug development. Brusatol is a new and effective antimalarial drug, which acts on GSK3 3 , ATP6A, ATP6B, ATP6M, MSP-2, EMP1, AMA-1, CTCS and many other proteins.

\section{Conclusion}

This study showed that the $2 \mathrm{mg} / \mathrm{kg}$ brusatol was effective on the inhibition of the growth of parasitemia in $P$. berghei-infected mice and there was little but reversible effect on host liver and kidney function.

Brusatol could play an effective role in treating malaria by regulating the host expression of TNF - $a$, IFN- $\gamma$, IL-4. RNA-seq results showed that the gene expression of $P$. berghei changed a lot after brusatol treatment, and there were 388 up-regulated genes and 424 down-regulated genes. Among those, GSK3 $\beta$, ATP6A, ATP6B, ATP6M, MSP-2, EMP1, AMA-1, CTCS, and other proteins might be the antimalarial targets.

\section{Declarations}

\section{Ethics approval and consent to participate}

The animal study was reviewed and approved by Institutional Animal Care and Use Committee of the Dalian Medical University in Dalian, China.

\section{Consent for publication}

No applicable

\section{Availability of data and materials}

The original contributions presented in the study are included in the article, further inquiries can be directed to the corresponding author/s.

\section{Competing interests}

The authors declare that they have no competing interests. 


\section{Funding}

This work was supported by Liaoning Provincial Program for Top Discipline of Basic Medical Sciences and Innovative Experiment Projects for University Students of Dalian Medical University (x201910161146).

\section{Author Contributions}

Yuanhua Qin conceived the project. Yixin Ren, Shanshan Xu, Yuan Gao performed animal experiments, and analyzed the results. Yufeng Chen, Xiaodong Dai, Lei Shi, Feng Zhao provided key technical support. Yuanhua Qin wrote the manuscript with input from all authors.

\section{Acknowledgement}

We thank Weifeng Mao for proofreading the entire manuscript.

\section{Authors' information}

1. College of Basic Medical Sciences, Dalian Medical University, Dalian 116044, Liaoning, China. 2. 2017 Clinical Medicine (Second Affiliated Hospital), Dalian Medical University, Dalian, China. 3. Department of Microorganism Examination, Dalian Center for Disease Control and Prevention, Dalian, China.

\section{References}

1. World Health Organization, World Malaria Report 2019,World Health Organisation, Geneva, Switzerland. 2019, https://www.who.int/publications-detail/world-malaria-report-2019.

2. Harald Noedl Y, Se K, Schaecher BL, Smith D, Socheat, Mark M, Fukuda. Evidence of artemisinin resistant malaria in western Cambodia [J]. N Engl J Med. 2008;359(24):2619-20.

3. Rick M, Fairhurst, Arjen M, Dondorp. Artemisinin-resistance Plasmodium falciparum malaria[J]. Microbiol Spectr. 2016;4(3):1-16.

4. Chanaki Amaratunga S, Sreng S, Suon ES, Phelps K, Stepniewska P, Lim, et al. Artemisinin-resistant Plasmodium falciparum in Pursat province, western Cambodia: a parasite clearance rate study. Lancet Infect Dis. 2012;12(11):851-8.

5. Phyo AP, Nkhoma S, Stepniewska K, Ashley EA, Nair S, McGready R, et al. Emergence of artemisininresistant malaria on the western border of Thailand: a longitudinal study[J]. Lancet. 2012;379(9830):1960-6. 
6. Hien TT, Thuy-Nhien NT, Phu NH, Maciej F, Boni, Ngo Viet Thanh, Nguyen Thuy Nha-Ca, et al. In vivo susceptibility of Plasmodium falciparum to artesunate in Binh Phuoc Province, Vietnam[J]. Malar J, 2012, 11: 355.

7. Aline Uwimana E, Legrand BH, Stokes J-LM, Ndikumana M, Warsame N, Umulisa, et al. Emergence and clonal expansion of in vitro artemisinin-resistant Plasmodium falciparum kelch13 R561H mutant parasites in Rwanda. Nat Med. 2020;26(10):1602-8.

8. Kim HS, Shibata Y, Ko N, Ikemoto N, Ishizuka Y, Murakami N, et al. Potent in vivo antimalarial activity of 3,15-di-O-acetylbruceolide against Plasmodium berghei infection in mice. Parasitol Int. 2000;48(3):271-4.

9. Lee KH, Tani S, Imakura Y. Antimalarial agents. Synthesis of a brusatol analog and biological activity of brusatol-related compounds. J Nat Prod. 1987;50(5):847-51.

10. O'Neill MJ, Bray DH, Boardman P, Chan KL, Phillipson JD, Warhurst DC, et al. Plants as sources of antimalarial drugs, Part 4: Activity of Brucea javanica fruits against chloroquine-resistant Plasmodium falciparum in vitro and against Plasmodium berghei in vivo. J Nat Prod. 1987;50(1):41-8.

11. O'neill MJ, Bray DH, Boardman P, Phillipson JD, Warhurst DC. Plants as Sources of Antimalarial Drugs Part. 1. In vitro Test Method for the Evaluation of Crude Extracts from Plants. Planta Med. 1985;51(5):394-8.

12. Pavanand K, Nutakul W, Dechatiwongse T, Pavanand K, Nutakul W, Dechatiwongse T, Yoshihira K, Yongvanitchit K, Scovill JP, et al. In vitro antimalarial activity of Brucea javanica against multi-drug resistant Plasmodium falciparum. Planta Med, 1986, (2):108-111.

13. Guru PY, Warhurst DC, Harris A, Phillipson JD. Antimalarial activity of bruceantin in vitro. Ann Trop Med Parasitol. 1983;77(4):433-5.

14. Straimer J, Gnädig NF, Witkowski B, Amaratunga C, Duru V, Ramadani AP. Drug resistance. K13propeller mutations confer artemisinin resistance in Plasmodium falciparum clinical isolates. Science. 2015;347(6220):428-31.

15. Hanna M, Leinonen E, Kansanen P, Pölönen M, Heinäniemi. Anna-Liisa Levonen. Role of the Keap1Nrf2 pathway in cancer[J]. Adv Cancer Res. 2014;122(4):281-320.

16. Ryoo I-G, Ha H, Kwak M-K. Inhibitory Role of the KEAP1-NRF2 Pathway in TGFß1-Stimulated Renal Epithelial Transition to Fibroblastic Cells: A Modulatory Effect on SMAD Signaling[J]. Plos One. 2014;9(4):e93265.

17. Lee JH, Rangappa S, Mohan CD, Gautam B, Sethi Z-X, Lin, Kanchugarakoppal S, Rangappa, et al. Brusatol, a Nrf2 Inhibitor Targets STAT3 Signaling Cascade in Head and Neck Squamous Cell Carcinoma[J]. Biomolecules. 2019;9(10):550-.

18. Peter W, Portus H, Robinson L. The four-day suppressive in vivo antimalarial test[J]. Pathogens Global Health. 1975;69:155-71.

19. Wang S, Dong Y. Research progress on the role of cytokines in plasmodium infection. Chinese Journal of Zoonoses. 2012;28(1):84-7. 
20. Shear HI, Srinivasan R, Nolan T, Ng C. Role of IFN-yin lethal and non-lethal malaria in susceptible and resistant murine hosts [J]. The Journal of immunology,1989,143(6):2038-2044.

21. Yingjie Liu Y, Cao J, Wang E, Luo S, Zhang. Experimental studies in vitro on no synthesis of macrophages induced by IFN-yduring the infection of plasmodium yoelii. Acta Parasitologica et Medica Entomologica Sinica. 2002;9(2):70-3.

22. Su Z, Stevenson MM. Central Role of Endogenous Gamma Interferon in Protective Immunity against Blood-Stage Plasmodium chabaudi AS Infection[J]. Infect Immun. 2000;68(8):4399-406.

23. Sandrine Cabantous B, Poudiougou A, Traore M, Keita MB, Cisse O, Doumbo, et al. Evidence That Interferon-y Plays a Protective Role during Cerebral Malaria. J Infect Dis. 2005;192(5):854-60.

24. DeKossodo S, Grau GE. Profiles of cytokine production in relation with susceptibility to cerebral malaria[J]. The Journal of Immunology,1993,151(9):4811-4820.

25. Leanne J, Robinson, Marthe C, D'Ombrain DI, Stanisic J, Taraika N, Bernard, Jack S, Richards, et al. Cellular tumor necrosis factor, gamma interferon,and interleukin-6 responses as correlates of immunity and risk of clinical plasmodium falciparum malaria in children from Papua New Guinea[J]. Infect Immun. 2009;77(7):3033-43.

26. Kumaratilake LM, Ferrante A. IL-4 inhibits macrophage-mediated killing of Plasmodium falciparum in vitro. A possible parasite-immune evasion mechanism. J Immunol. 1992;149(1):194-9.

27. John M, Ong'echa, Allison M, Remo J, Kristoff JB, Hittner T, Were $C$, Ouma, et al. Increased circulating interleukin (IL)-23 in children with malarial anemia: In vivo and in vitro relationship with co-regulatory cytokines IL-12 and IL-10[J]. Clinical Immunology. 2008;126(2):211-21.

28. Troye-Blomberg M, Sjöholm PE, Perlmann H, Patarroyo ME, Perlmann P. Regulation of the immune response in Plasmodium falciparum malaria. I. Non-specific proliferative responses in vitro and characterization of lymphocytes. Clinical Experimental Immunology. 1983;53(2):335-44.

29. Masch A, Kunick C. Selective inhibitors of Plasmodium falciparum glycogen synthase-3 (PfGSK-3): New antimalarial agents[J]. Biochim Biophys Acta. 2015;1854(10 Pt B):1644-9.

30. Jung M, Kim H, Nam KY. Kyoung Tai No. Three-dimensional structure of Plasmodium falciparum Ca2+-ATPase (PfATP6) and docking of artemisinin derivatives to PfATP6[J]. Bioorganic \& Medicinal Chemistry Letters, 2005, 15(12):pp. 2994-7.

31. Krishna S, Pulcini S, Fatih F, Staines H. Artemisinins and the biological basis for the PfATP6/SERCA hypothesis[J]. Trends in Parasitology. 2010;26(11):517-23.

32. Uhlemann AC, Krishna S. Antimalarial Multi-Drug Resistance in Asia: Mechanisms and Assessment[J]. Curr Top Microbiol Immunol. 2005;295:39-53.

33. Snounou G, Zhu X, Siripoon N, Jarra W, Thaithong S, Brown KN, et al. Biased distribution of msp1 and msp2 allelic variants in Plasmodium falciparum populations in Thailand[J]. Transactions of the Royal Society of Tropical Medicine \& Hygiene, 1999, 93(4):pp. 369-74.

34. Hussein Mohammed T, Mindaye M, Belayneh M, Kassa A, Assefa M, Tadesse, et al. Genetic diversity of Plasmodium falciparum isolates based on MSP-1 and MSP-2 genes from Kolla-Shele area, Arbaminch Zuria District, southwest Ethiopia[J]. Malaria Journal. 2015;14:73. 
35. Anyirékun F, Somé T, Bazié I, Zongo RS, Yerbanga F, Nikiéma C. Neya, et al. Plasmodium falciparum msp1 and msp2 genetic diversity and allele frequencies in parasites isolated from symptomatic malaria patients in Bobo-Dioulasso, Burkina Faso[J]. Parasites \& Vectors, 2018, 11(1):p. 323.

36. Turner L, Lavstsen T, Mmbando BP. IgG antibodies to endothelial protein C receptor-binding cysteinerich interdomain region domains of Plasmodium falciparum erythrocyte membrane protein 1 are acquired early in life in individuals exposed to malaria. Infect Immun. 2015;83(8):3096-103.

37. Bull PC, Abdi Al. The role of PfEMP1 as targets of naturally acquired immunity to childhood malaria: prospects for a vaccine. Parasitology. 2016;143(2):171-86.

38. Hsieh F-L, Turner L, Bolla JR, Robinson CV, Lavstsen T, Matthew K, Higgins. The structural basis for CD36 binding by the malaria parasite[J]. Nat Commun. 2016;7:12837.

39. Damien R, Drew PR, Sanders G, Weiss PR, Gilson, Brendan S, Crabb JG, Beeson. Functional Conservation of the AMA1 Host-Cell Invasion Ligand Between P. falciparum and P. vivax: A Novel Platform to Accelerate Vaccine and Drug Development[J]. The Journal of Infectious Diseases J Infect Dis. 2018;217(3):498-507.

\section{Figures}

Fig. 1

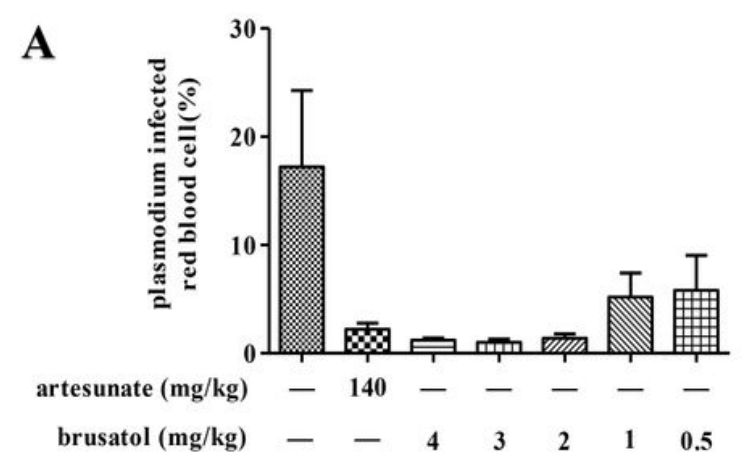

C

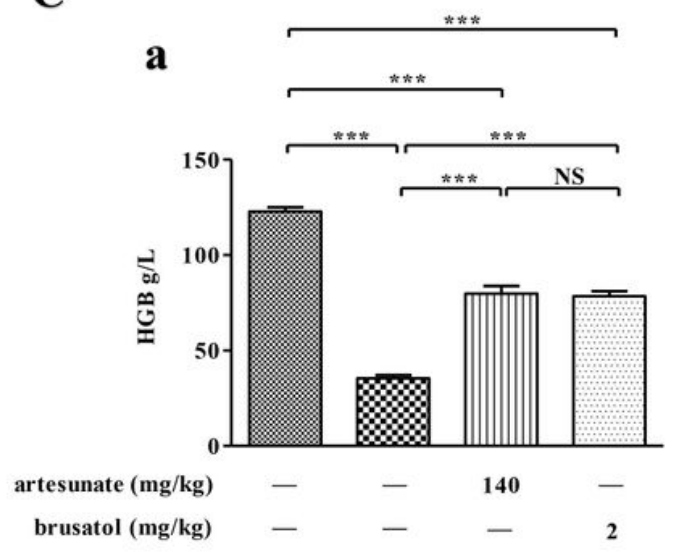

B
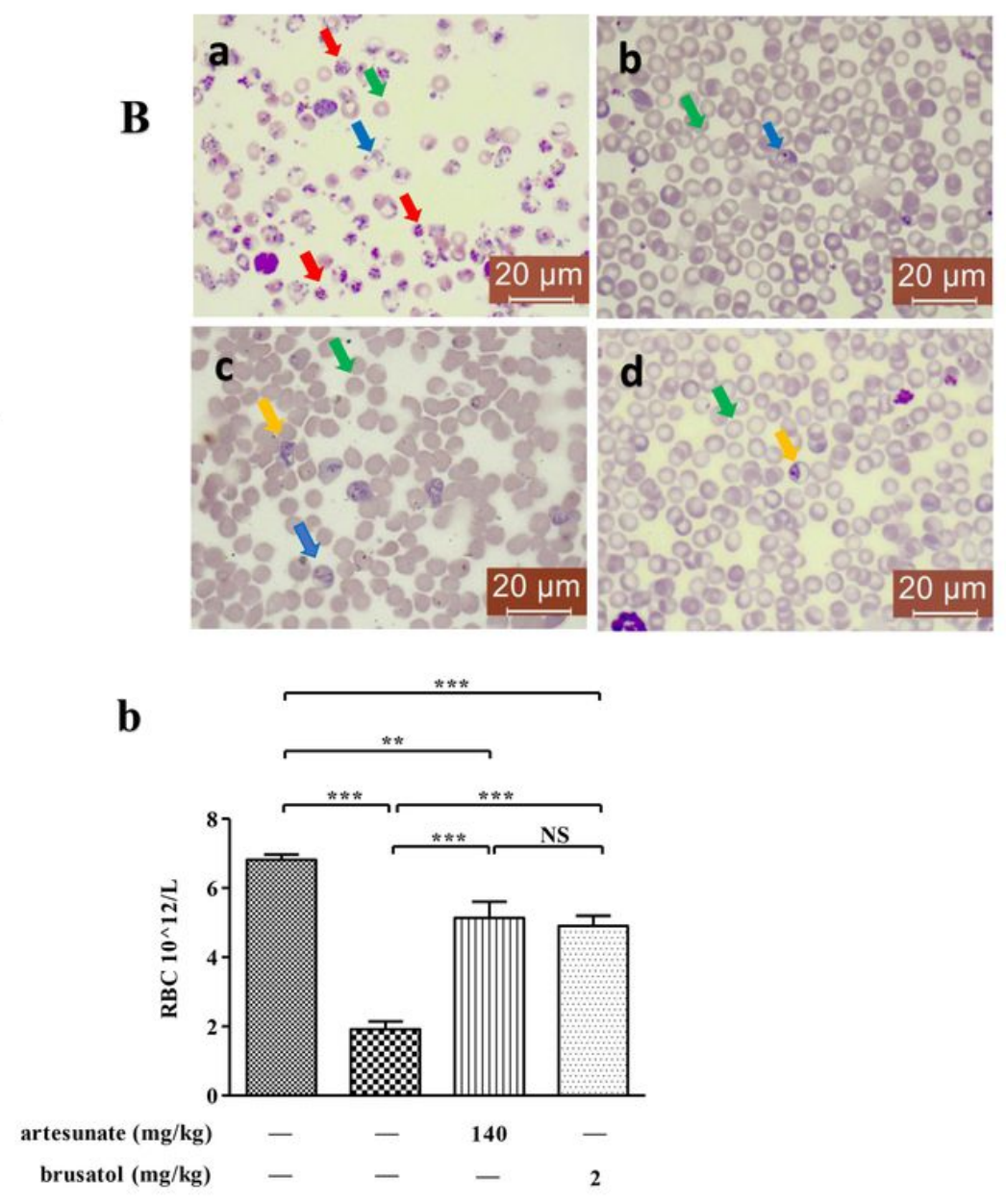
Effect of different doses of brusatol and artesunate on parasitemia in P. berghei-infected mice. A: The percentage of plasmodium infected red blood cells after different treatments. B: Observation of plasmodium infected red blood cells after different treatments under microscope (a: control, parasitemia was near $17 \%$; b: artesunate of $140 \mathrm{mg} / \mathrm{kg}$, parasitemia was near $2 \%$; c: brusatol of $0.5 \mathrm{mg} / \mathrm{kg}$, parasitemia was near $6 \%$; $\mathrm{d}$ : brusatol of $2 \mathrm{mg} / \mathrm{kg}$, parasitemia was near $1 \%$ ). The red arrows refer to the schizont stage; the yellow arrows refer to the trophozoite stage; the blue arrows refer to the ring stage; the green arrows refer to normal red blood cells. C: The HGB and RBC statistic of normal, control, $140 \mathrm{mg} / \mathrm{kg}$ artesunate and $2 \mathrm{mg} / \mathrm{kg}$ brusatol groups. Asterisks $\left(^{*}\right)$ indicate significant differences between groups ( ${ }^{*}<<0.05,{ }^{*} \mathrm{P}<0.01,{ }^{*} * \mathrm{P}<0.001$ vs. adjacent). NS indicates no statistical differences between groups.
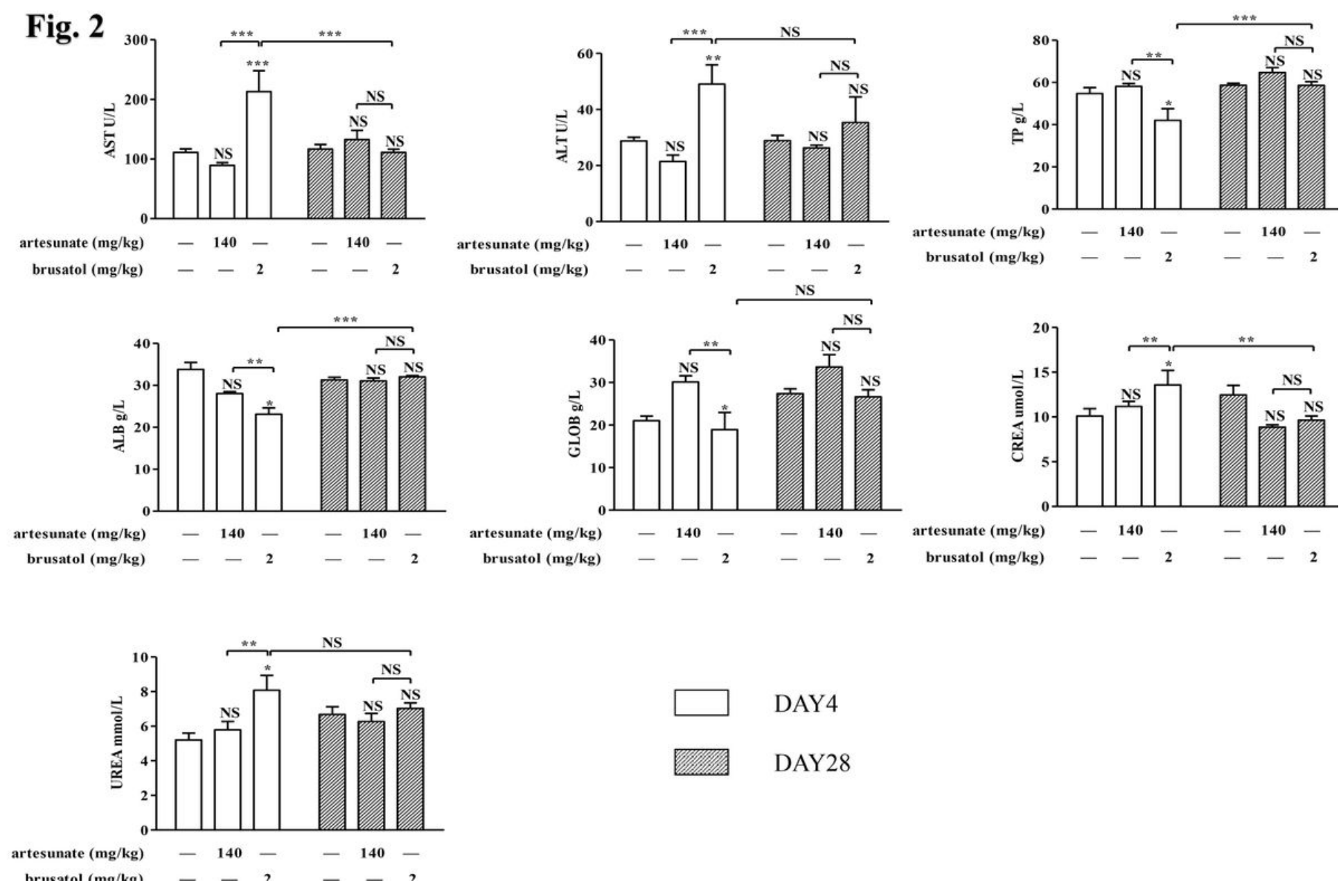

\section{Figure 2}

Changes of the serum concentrations of AST, ALT, TP, ALB, GLOB, CREA and UREA in 3 groups (control, artesunate and brusatol) on Day4 and Day28. Asterisks $(*)$ indicate significant differences between groups ( ${ }^{*} P<0.05,{ }^{*} P<0.01,{ }^{*} * \mathrm{P}<0.001$ vs. adjacent). NS indicates no statistical differences between groups. Note: AST: aspartate transaminase; ALT: alanine transaminase; TP: total protein; ALB: albumin; GLOB: globulin; CREA: creatinine; UREA: urea 
Fig. 3

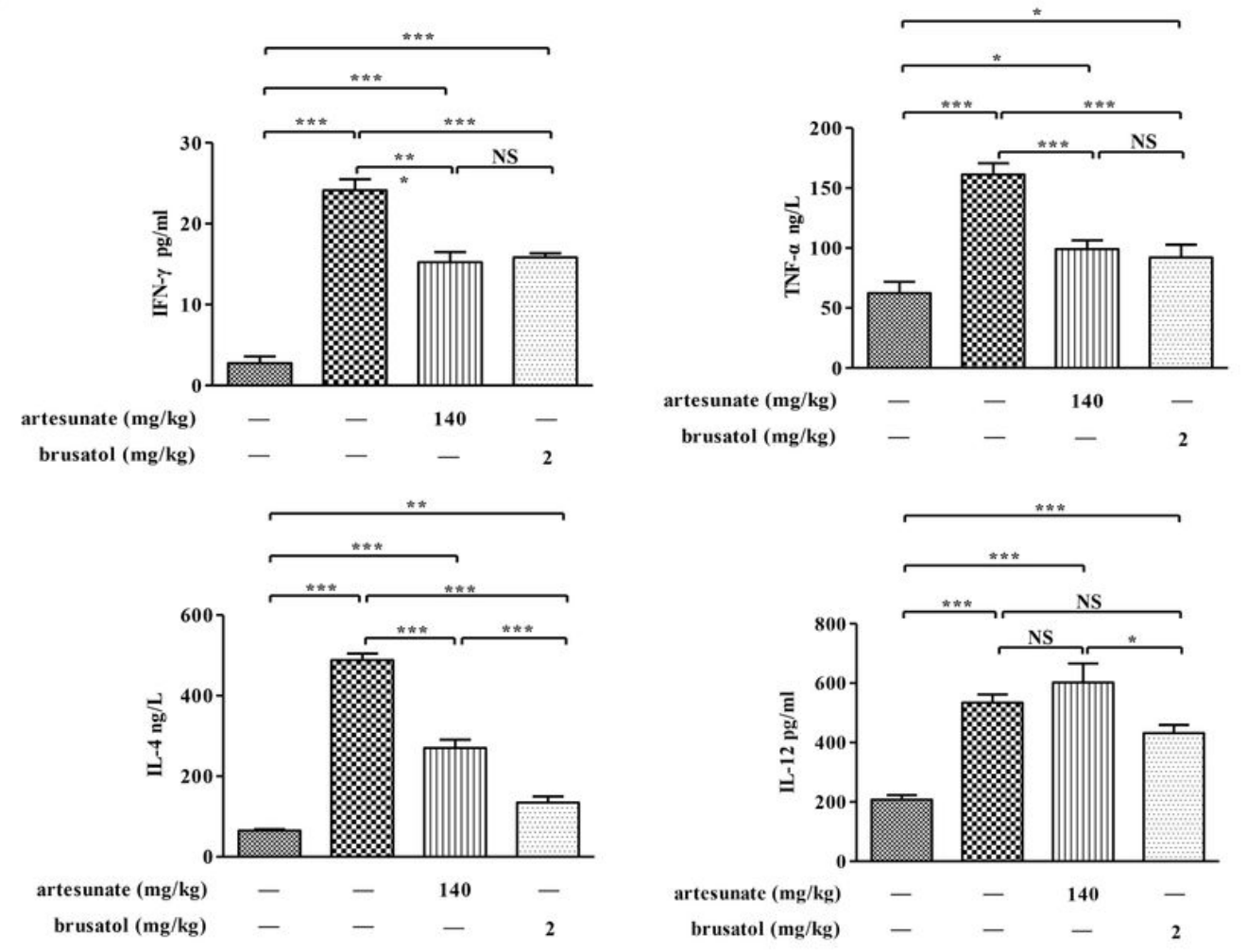

Figure 3

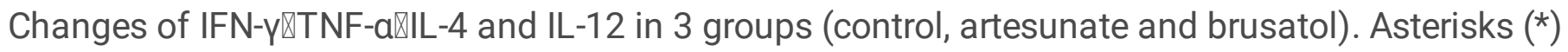
indicate significant differences between groups $\left({ }^{\star} P<0.05\right.$, ${ }^{\star *} P<0.01$, ${ }^{\star \star \star} P<0.001$ vs. adjacent). NS indicates no statistical differences between groups. 
Fig. 4

A

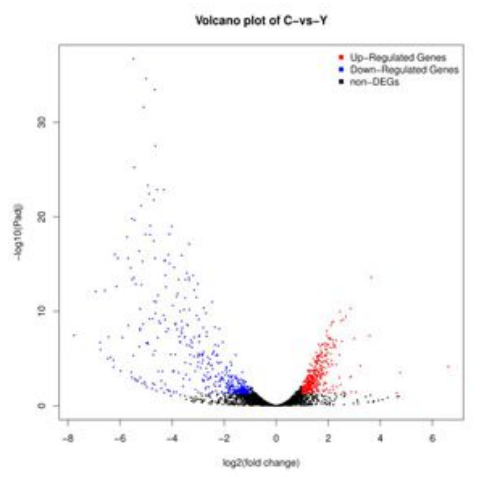

B

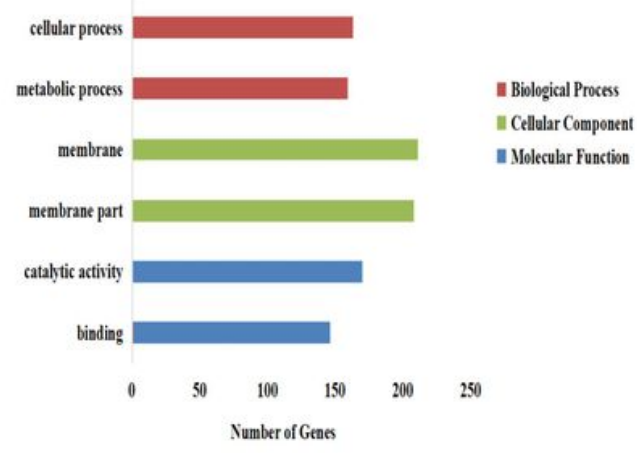

C

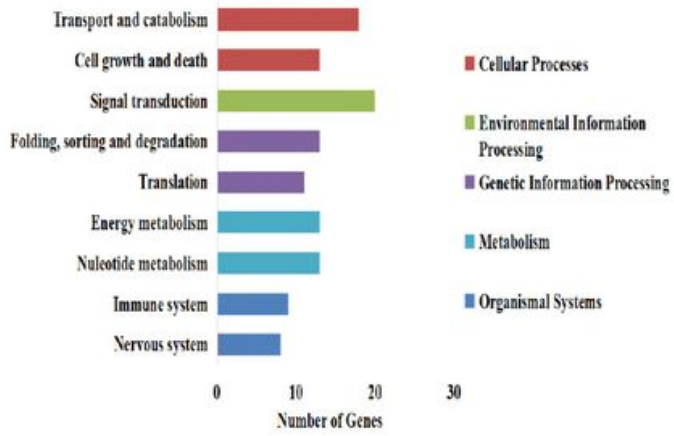

\section{Figure 4}

GO enrichment and KEGG pathway analyses of the DEGs. A: Volcano map of the DEGs between brusatol and control groups. Red dots represent upregulated genes, blue dots represent downregulated genes and black dots represent unchanged genes. B: GO enrichment analyses on biological processes, cellular components, and molecular functions of the DEGs between brusatol and control groups. C: KEGG pathway analysis of the DEGs between brusatol and control groups. 
Fig. 5

A
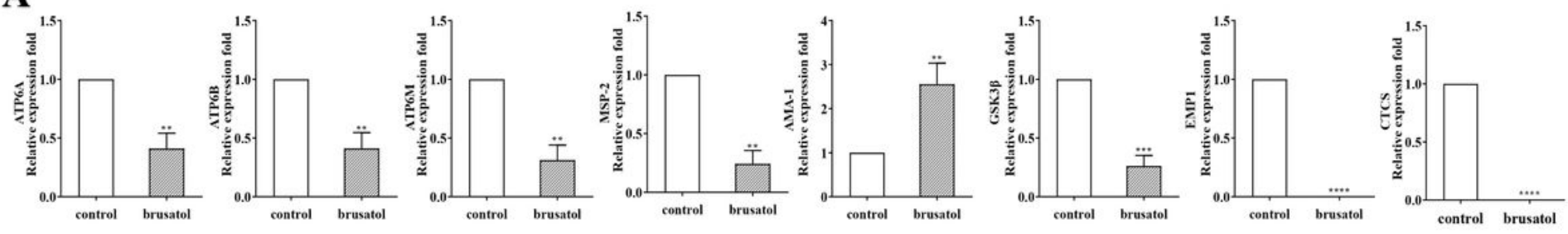

B
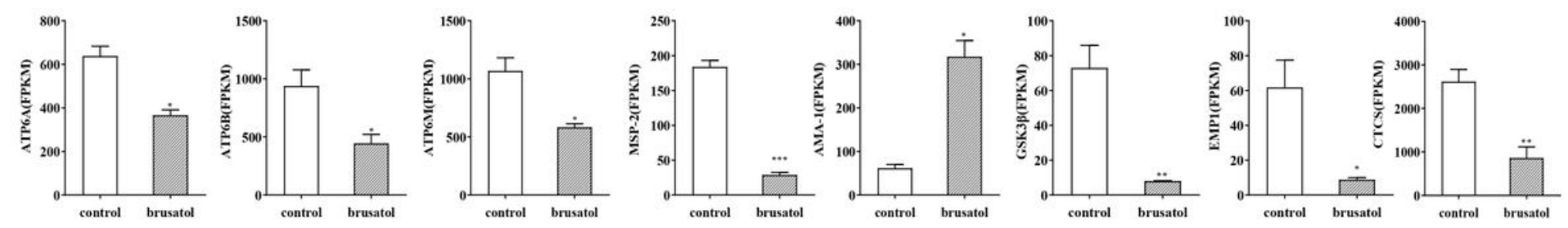

\section{Figure 5}

Expression levels of ATP6A, ATP6B, ATP6M, MSP-2, AMA-1, GSK3 3 , EMP1, CTCS between control, and brusatol groups. A: Relative mRNA expression levels of selected genes between two groups determined via reverse transcription-quantitative PCR analysis. B: mRNA expression levels of selected genes between two groups according to the database and its unit of measurement is FPKM. FPKM, Fragments Per Kilobase per Million. 